\title{
Taxonomía y dinámica de las expectativas económicas de los empresarios industriales en Uruguay. Un análisis de conglomerados
}

\author{
Recibido: 11 de octubre de 2013 - Aceptado: 30 de septiembre de 2014 \\ Doi: dx.doi.org/10.12804/rev.econ.rosario.17.02.2014.02
}

\author{
Bibiana Lanzilotta Mernies* \\ Centro de Investigaciones Económicas (CINVE)
}

\section{Resumen}

Este artículo investiga los vínculos existentes entre los sectores industriales en Uruguay, con el fin de extraer señales acerca del proceso de formación de sus expectativas. El enfoque es predominantemente empírico, basado en la exploración de algoritmos de agrupación, mediante una única conexión con el vecino más cercano. Los resultados muestran que existe un grupo de industrias conectadas en expectativas, mayormente integrado por aquellas más expuestas a la competencia internacional. Estas ocupan los nodos centrales de la red de conexiones intersectoriales. Adicionalmente, se prueba que la cohesión entre las ramas industriales tiene una correlación positiva con el crecimiento manufacturero.

Clasificación JEL: C14, D71, D84

Palabras clave: expectativas, análisis de clusters, clubes.

* Centro de Investigaciones Económicas. Dirección: Uruguay 1242, Montevideo, Uruguay. Teléfono: (598) 29081533. Correo electrónico: bibiana@cinve.org.uy

Para citar este artículo: Lanzilotta, M. B. (2014). Taxonomía y dinámica de las expectativas económicas de los empresarios industriales en Uruguay. Un análisis de conglomerados. Revista de Economía del Rosario, 17(2), 229-256. doi: dx.doi.org/10.12804/rev.econ.rosario.17.02.2014.02 


\title{
Taxonomy and Dynamic of Entrepreneurial Expectations in Uruguay. A Cluster Analysis
}

\begin{abstract}
This paper investigates the links between industrial sectors in Uruguay, in order to extract signals about their expectation process formation. The approach is predominantly empirical, based on clustering algorithms with a single connection to the nearest neighbor. The results show that there is a group of connected industries in expectations, mostly composed of those most exposed to international competition. They hold the central nodes of the network of cross connections. Additionally, it is shown that the cohesion between industries is positively correlated with manufacturing growth.
\end{abstract}

JEL Classification: C14, D71, D84

Keywords: expectations, cluster analysis, clubs.

\section{Taxonomia e dinámica das expectativas econômicas dos empresários industriais no Uruguai. Uma análise de conglomerados}

\begin{abstract}
Resumo
Este artigo pesquisa os vínculos existentes entre os sectores industriais no Uruguai com o objetivo de extrair sinais acerca do processo de formação de suas expectativas. O enfoque é predominantemente empírico, baseado na exploração de algoritmos de agrupação mediante uma única conexão com o vizinho mais próximo. Os resultados mostram que existe um grupo de indústrias conectadas em expectativas, maiormente integrado por aquelas mais expostas à concorrência internacional. Estas ocupam os nodos centrais da rede de conexões intersetoriais. Adicionalmente, prova-se que a coesão entre os ramos industriais tem uma correlação positiva com o crescimento manufatureiro.
\end{abstract}

Classificação JEL: C14, D71, D84

Palavras-chave: expectativas, Análise de clusters, Clubes. 


\section{Introducción}

El problema de cómo las personas se forman expectativas y cómo interactúan con la estructura institucional de la economía es una cuestión que ha despertado históricamente el interés de los investigadores (Rosser, 2001).

En este trabajo se intenta aportar nuevos elementos a esta discusión al estudiar empíricamente la relación entre el ciclo económico y la formación de expectativas por parte del sector empresarial. Este artículo indaga sobre la existencia de clusters en expectativas sobre el futuro de la economía por ramas de actividad económica. De este modo, se construye una taxonómica de la industria y se identifica si la aglomeración de expectativas responde o depende de la fase del ciclo económico, es decir, si varía cuando la economía está en recesión o en el auge del ciclo.

La literatura en economía ha destacado históricamente, desde distintas ópticas, el papel de las interacciones entre los agentes en la formación de expectativas. La dependencia de las personas respecto de las otras en la formación de sus expectativas abre la posibilidad, por ejemplo, de comportamientos asociados con los cambios en masa o las profecías autocumplidas. En contextos de incertidumbre (y asimetría de información), las personas adoptan convenciones y en tales circunstancias los intentos por predecir el comportamiento de los demás agentes cumple un papel crucial. A su vez, los enfoques poskeynesianos han propuesto diferentes hipótesis sobre la formación de expectativas, lo que pone énfasis en la naturaleza de la interacción de las personas y en diversas complejidades de la dinámica de grupos. Autores como Carabelli (1988), Davis (1993) y Arestis (1996) perciben el problema de la incertidumbre de los agentes sobre las expectativas de los demás como la fuente fundamental de la incertidumbre general. Grandmont (1998) sugiere que cuando los agentes enfrentan algún tipo de incertidumbre acerca de la dinámica del sistema económico o social, el proceso de aprendizaje puede generar inestabilidad local y expectativas autocumplidas (regularidad a la que denomina principio de incertidumbre).

En ese marco, los enfoques que sostienen que las expectativas son el resultado de la racionalidad limitada y de las convenciones han ido ganando terreno crecientemente en contra de la extrema simpleza de la hipótesis de expectativas racionales (Kahneman, Slovic \& Tversky, 1982; Thaler, 1994). En particular, estos enfoques proponen que los agentes aprenden de la conducta y las actitudes de las personas de su entorno y de los fenómenos más sobresalientes que captan su atención.

Por su parte, Potts (2000), desde una óptica evolucionista, propone una particular visión respecto del proceso de formación de expectativas de los agentes, donde el contexto y la interacción entre los agentes tienen un rol particular. Su 
concepción sobre la dinámica de formación de expectativas se basa en la noción de agentes complejos y parcialmente especializados que construyen modelos internos a partir del ambiente externo, situados en un contexto de red.

Tanto la literatura sobre gestión y dirección de empresas y marketing, como la de innovación y cambio técnico han avanzado hacia una concepción de redes (Roy, Sivakumar \& Wilkinson, 2004). Aquí, el flujo de información y conocimiento es uno de los elementos más relevantes que caracterizan a la red. En este sentido, Sorenson, Rivkin y Fleming (2006) sostienen que la mayor proximidad entre los agentes favorece la transmisión de conocimientos, en particular cuando estos son medianamente complejos.

La investigación empírica sobre expectativas de los agentes es limitada en Uruguay. Algunos de los estudios disponibles tienen como objeto de análisis las expectativas de inflación de los analistas que se recogen en las encuestas que realiza el Banco Central (Borraz \& Gianelli, 2010; Lanzilotta, Fernández \& Zunino, 2008; Zunino, Lanzilotta \& Fernández, 2010). Por otra parte, en Lanzilotta (2006) se investiga sobre las expectativas de los empresarios y su vínculo con la actividad económica desde una perspectiva macroeconómica. A su vez, en Lanzilotta (2014) se indaga sobre la dinámica de la transmisión de los shocks sobre expectativas dentro de la industria, destacándose el papel que tienen las industrias exportadoras y las productoras de bienes sustitutivos de importaciones.

El presente trabajo continúa con esa línea de investigación e indaga sobre la existencia - dentro de la industria manufacturera uruguaya- de clusters o clubes integrados por ramas que posean similares expectativas sobre el futuro de la economía. A partir del análisis estadístico de las expectativas declaradas por los empresarios, se procura develar una particular faceta de la taxonomía de la industria e identificar las ramas que cumplen el rol de núcleos, ya que aglomeran a un conjunto significativo de otras ramas en su entorno. Adicionalmente, sobre la base de series temporales construidas a partir de las distancias mínimas entre las expectativas de los sectores industriales, se explora el vínculo entre su grado de cohesión, el contexto económico general y el desempeño de la industria manufacturera en particular.

El análisis toma como referencia a las expectativas declaradas por los empresarios, consideradas a nivel de ramas (4 dígitos) sobre un fenómeno común: el contexto económico nacional. Los datos surgen de la Encuesta Mensual Industrial (EMI) que elabora la Cámara de Industrias del Uruguay (CIU), entre los años 1998 y 2011.

El análisis empírico evita limitarse a un esquema teórico que restrinja a priori la identificación de los vínculos. Se utiliza un procedimiento estadístico no paramétrico que combina un conjunto de herramientas metodológicas, basado en 
artículos de Brida \& Punzo (2003), Brida, Puchet \& Punzo (2003), Brida \& Risso (2007), Brida, Matesaenz \& Risso (2009), Brida, London \& Risso (2010), entre otros.

Precisamente, la conjunción de la metodología empírica y del objeto de estudio y las expectativas empresariales constituyen la originalidad de este trabajo. Si bien, existen numerosas investigaciones que aplican a diversas temáticas de esta combinación de metodologías estadísticas, ninguno se conoce aplicado a indicadores de expectativas.

El resto del documento se estructura como sigue. En la próxima sección se exponen las ventajas e inconvenientes, así como las precauciones al utilizar las encuestas cualitativas sobre expectativas, se presentan algunos antecedentes al respecto. En la tercera sección se explica la metodología y en la cuarta, la estrategia empírica. En la quinta sección se describe la taxonomía de la industria de acuerdo con las expectativas y en la sexta, los resultados hallados respecto de la conexión entre expectativas y desempeño. Finalmente, la séptima sección reúne las conclusiones finales.

\section{El uso de encuestas de expectativas en el análisis económico}

El empleo de indicadores que dan cuenta de las expectativas de los agentes recabadas en encuestas es extendido en países que cuentan con un sistema de estadísticas desarrollado. Este tipo de indicadores ha sido largamente utilizado en la literatura aplicada para captar y anticipar los movimientos de diversas variables, como las tasas de interés, el desempleo o los precios. También han sido empleados para arrojar luz sobre la formación de expectativas y los planes de las empresas. Los estudios empíricos basados en encuestas sobre expectativas buscan, generalmente, indagar acerca de su naturaleza y el proceso de formación en la práctica, así como explorar su potencial para mejorar el funcionamiento de los métodos de predicción convencionales (véase Pesaran \& Weale, 2006, para una revisión de esta literatura).

Numerosos estudios emplean expectativas cuantitativas con fines de pronóstico y detección de cambios en las fluctuaciones cíclicas (Batchelor, 1982; Berk, 1999; Hanssens \& Vanden, 1987; Kangasniemi Kangassalo \& Takala, 2010; Kangasniemi \& Takala, 2012; Kauppi, Lassila \& Teräsvirta, 1996; Öller, 1990; Rahiala \& Teräsvirta, 1993; Smith \& McAleer, 1995; Svensson, 1997). Entre los que exploran los datos de las encuestas de opinión como indicadores del comportamiento empresarial, para testear diferentes esquemas de formación de expectativas o interacciones entre agentes, pueden citarse a Batchelor (1982), Smith \& McAleer (1995) y Alfarano \& Milakovic (2010), entre otros. 
Varios problemas han sido señalados en cuanto al tratamiento e interpretación de los datos de estas encuestas. Pesaran \& Weale (2006) señalan dos aspectos clave a considerar en el tratamiento agregado de los datos de expectativas con fines de la predicción macro y su modelización: la forma en que se recogen las respuestas y la metodología de conversión en datos cuantitativos agregados. Respecto del primer grupo, uno de los problemas más importantes es que los resultados de las encuestas son sensibles a errores de muestreo y la formulación de las preguntas (Chan-Lee, 1980). Por otra parte, los encuestados pueden expresar opiniones que son diferentes de las que inspiran finalmente sus acciones y podrían tratar de manipular las respuestas para evitar el suministro de datos confidenciales o para inducir el resultado deseado (Nardo, 2003). Otro de los problemas refiere al asunto de encuadre que puede introducir sesgos en las respuestas.

A nivel internacional, los intentos para comparar y analizar las ventajas e inconvenientes de los diferentes métodos de cuantificación no tienen demasiados ejemplos en la literatura. Common (1985) es uno de ellos, y concluye que ningún método parece preferible a otro. Nardo (2003) y Nardo \& Cabeza-Gutés (2009) encuentran que el método tiene un desempeño medio (no obstante, sus conclusiones quedan relativizadas al proceso generador de datos elegido para sus simulaciones).

¿En qué medida los problemas antes señalados están presentes en esta investigación empírica sobre expectativas en Uruguay? Los datos de expectativas empresariales que se emplean en este artículo surgen de una encuesta a industriales de larga data, llevada a cabo por la Cámara de Industrias del Uruguay (CIU) desde 1997 a la fecha. En esa encuesta, además de relevarse las ventas, se indaga acerca de las expectativas sobre la evolución de la actividad de la propia empresa, del mercado externo e interno, de la rama de actividad industrial en la que se desempeña y de la economía en su conjunto. Los indicadores de expectativas que se estudian en este trabajo refieren a esta última dimensión. ${ }^{1}$

Respecto de la conversión de los datos cualitativos a una medida cuantitativa de expectativas, se empleó aquí la estadística de Balance. Esta estadística es ampliamente utilizada (en particular por Eurostat) por la simplicidad y claridad en su interpretación. Según esta, los indicadores cuantitativos de expectativas

1 Como forma de examinar la representatividad de la encuesta que lleva a cabo la CIU, se compararon las estadísticas de ventas que surgen de esta encuesta con la serie que releva el organismo oficial de estadísticas en Uruguay. Se verificó que la evolución de las ventas industriales relevadas por la CIU acompasa razonablemente la que publica el Instituto Nacional de Estadística, lo cual permite suponer que la encuesta de expectativas posee una razonable representatividad. 
se construyen como el cociente entre la suma de las respuestas positivas y las negativas y el total de respuestas. Cada respuesta se incorpora al indicador con el mismo peso, independiente del tamaño de la empresa o de la rama. No obstante, cabe señalar que los resultados a los que se arriba en este estudio pueden estar sesgados por la particular elección de la metodología de conversión de datos. ${ }^{2}$

\section{Metodología empírica}

El trabajo empírico se basa en la exploración de algoritmos de agrupación, mediante una única conexión con el vecino más cercano. Esta metodología sigue a la empleada por Brida, Parte, Risso \& Such (2010), Brida, London \& Risso (2010), Brida \& Risso (2007), basados en Mantegna (1999).

El método de agrupación se basa en el cálculo de distancias entre individuos (que en este caso son las ramas industriales). Estas distancias pueden establecerse en una dimensión simple o múltiple. En función del valor de la dimensión o variable, se obtiene una distancia métrica entre dos individuos (dos ramas). En este caso, la variable o dimensión es el valor promedio de las expectativas sobre la economía de los empresarios. La distancia $(d)$ que muestra el grado de coincidencia o lejanía entre los individuos respecto de sus expectativas se define en la ecuación (1):

$$
d(i, j)=\sqrt{\frac{\sum_{t=1}^{t=T}\left(i e c_{i t}-i e c_{j t}\right)^{2}}{T}},
$$

${\text { donde } i e c_{i t}}$ y $i e c_{j t}$ son los valores que toman los indicadores de los individuos $i$ y $j$ (ramas de la industria) en el momento $t$, respectivamente, mientras que $T$, es el total de periodos.

En función de estas distancias, se construyen los árboles de expansión mínima (Minimal Spanning Tree, MST) conectando a los individuos (ramas industriales) mediante el algoritmo Kruskal (1956). Este es un algoritmo aglomerativo de la teoría de grafos, que permite encontrar un árbol de expansión mínima en un grafo conectado y ponderado. El algoritmo busca el subconjunto de arcos (distancias entre los nodos o individuos), tal que incluya todos los individuos y cuya suma total sea mínima.

2 El empleo de otros métodos de conversión podría aportar robustez al análisis. 
El árbol se construye progresivamente, al asociar todos los individuos (ramas) de la muestra en un grafo caracterizado por la mínima distancia entre ellos, empezando por la más corta. Para una muestra de $n$ elementos o individuos el árbol que se obtiene es un grafo con $n-1$ conexiones. El algoritmo sigue los pasos detallados en Fung (2001) y en Brida, Matesanz \& Risso (2009).

La virtud del MST es que arroja un arreglo de los individuos (ramas), que contiene las conexiones más relevantes de cada elemento de la muestra (Mantegna, 1999). Dos vértices cualesquiera del MST se pueden conectar directamente o por medio de uno o más vértices. Estas conexiones representan los enlaces de mínima distancia entre estos. La idea básica consiste en elegir sucesivamente las aristas o arcos de mínimo peso, comenzando por la menor. Partiendo de una muestra de $n$ series de indicadores de expectativas (correspondiente a $n$ ramas), el algoritmo consiste en los siguientes pasos:

1) Se inicia el árbol MST con $n$ nodos y sin $\operatorname{arcos} \operatorname{MST}=(\{1,2, \ldots, n), \varnothing)$.

2) Se crea una lista de $\operatorname{arcos}(L)$, en orden ascendente de peso (en este caso, las distancias entre expectativas). Los arcos con el mismo peso son ordenados arbitrariamente.

3) Se selecciona el arco $(i, j)$ que esté al comienzo de la lista, se transfiere al MST y se borra de $L$.

4) Si $L$ es no vacío, se regresa al paso 3, de lo contrario se termina el proceso.

De esta forma, el MST permite identificar a los individuos (ramas) más conectados con el resto y a los más aislados. Este mismo procedimiento posibilita construir la matriz de distancia ultramétrica subdominante a partir del MST, que da cuenta del grado de organización jerárquica de los vértices del grafo. Esta matriz se obtiene a partir de la definición de la distancia ultramétrica $d<(i, j)$ entre $i$ y $j$, como el máximo valor de las distancias euclidianas $d_{m}(k, l)$ calculadas de moverse a un paso desde $i$ a $j$ por medio de la trayectoria más breve que conecta el vértice $i$ con el $j$ en el MST (Mantegna, 1999).

La distancia $d<(i, j)$ entre $i$ y $j$ está dada por:

$$
d<(i, j)=\operatorname{Max}\left\{d_{m}\left(w_{i} ; w_{i+1}\right) ; 1 \leq i \leq n-1\right\},
$$

donde $\left\{\left(\mathrm{w}_{1} ; \mathrm{w}_{2}\right),\left(\mathrm{w}_{2} ; \mathrm{w}_{3}\right), \ldots,\left(\mathrm{w}_{n-1}, \mathrm{w}_{\mathrm{n}}\right)\right\}$ denota la única trayectoria mínima en el MST que conecta $i$ y $j$, donde $w_{1}=i$ y $w_{n}=j$. De esta forma, se calcula el valor de $d<(i, j)$ para cada pareja de individuos (ramas industriales). A partir de estas, 
se construyen los árboles jerárquicos o dendrogramas Hierarchical Tree (HT). ${ }^{3}$ Estos muestran gráficamente los clusters de ramas industriales que pueden ser interpretados como clubes o grupos de comportamiento, respecto de las expectativas económicas. Un punto importante es la determinación del número de agrupamientos estadísticamente relevantes, en otras palabras, la identificación del mejor nivel para cortar el dendrograma. En este trabajo, la regla de detención empleada fue el pseudo $F$ ( $p s F)$, uno de los criterios más empleados usualmente en la literatura empírica.

\section{Estrategia empírica}

La unidad de análisis es cada una de las ramas del sector manufacturero uruguayo (4 dígitos de la Clasificación Industrial Internacional Uniforme, CIIU revisión 3), la variable o dimensión evaluada son las expectativas de los empresarios respecto de la economía.

El periodo de estudio abarca casi catorce años, y se utilizan datos mensuales provenientes de la encuesta industrial de la Cámara de Industrias del Uruguay, en lo que refiere a las variables de expectativas. Si bien, el análisis se extiende entre enero de 1998 y junio de 2011, debido a que no se contó con registros de la EMI para 4 meses, la muestra alcanzó a 158 observaciones por rama. La base de datos original fue depurada, ya que algunas series contenían vacíos de información, por lo que se consideraron solo 21 de las 35 ramas industriales relevadas. Cabe destacar que el conjunto de ramas consideradas representa alrededor de dos terceras partes del Valor Bruto de Producción del sector manufacturero uruguayo (si se excluye la refinería de petróleo).

Se consideraron por tanto las respuestas de 21 sectores industriales $(i: 1,2, \ldots$, 21 , ver descripción de las ramas en el anexo 2), durante 158 meses $(t: 1,2, \ldots, 158$; enero 1998 a junio 2011).

El indicador de expectativas sobre la economía (que se denota iec) se construye a partir de las respuestas cualitativas sobre las percepciones que poseen los empresarios, respecto de la evolución de la actividad económica del país seis meses adelante. La pregunta que se formula en la encuesta mensual ${ }^{4}$ es: "Considerando la situación actual, ¿cómo ve la evolución de la economía nacional,

3 La determinación del árbol jerárquico de una ultramétrica subdominante está completamente controlada por la matriz de distancia ultramétrica (Mantegna, 1999).

4 La encuesta revela información mensual de aproximadamente 250 firmas industriales. La metodología se puede consultar en: http:/ / www.ciu.com.uy/innovaportal/ file/15211/1/ metodologia_emi.pdf. 
de su sector y de su empresa en el horizonte de los próximos seis meses?". Las opciones de respuesta son mejor, peor o igual.

Para la agregación de los indicadores de expectativas a nivel de rama industrial, las respuestas positivas (espera mejora) se ponderan con +1 , las negativas (espera empeore) con $-1, y$ las restantes con 0 , sin sopesarlas por la participación de cada empresa en la producción de la respectiva rama. ${ }^{5}$ Así, el indicador de expectativas correspondiente a la rama $i$, se construye como:

$$
i e c_{i t}=\frac{\left(N u ́ m \_r e s p \_p o s i t_{i t}{ }^{*}(+1)+N u ́ m \_r e s p \_N e g_{i t}{ }^{*}(-1)+0 * N u ́ m \_R e s p \_I g u a l_{i t}\right)}{\text { Número total de respuestas mes } t}
$$

El rango de valores que toman estos indicadores es [-1,1].

La distancia entre las expectativas de las ramas $i, j$ en cada momento se calcula como:

$$
D_{t}\left(i e c_{i t}, i e c_{j t}\right)=\left(i e c_{i t}-i e c_{j t}\right)^{2}
$$

Esta podrá tomar valores entre 0 y 4 . La distancia máxima (expectativas opuestas) resulta de la combinación de: $i e c_{i t}=1$ y $i e c_{j t}=-1$. En tanto, la distancia mínima se da cuando los valores de los indicadores de una y otra rama en el momento $t$ son idénticos, es decir: $i e c_{i t}=i e c_{j ;}$, por lo que $D_{t}=0$.

La distancia de las expectativas entre los sectores $i, j$ a lo largo de $T$ se define como:

$$
d\left(\operatorname{Iec}_{i}^{T}, \operatorname{Iec}_{j}^{T}\right)=\sqrt{\frac{D_{1}\left(\operatorname{iec}_{i 1}, \operatorname{iec}_{j 1}\right)+D_{2}\left(\operatorname{iec}_{i 2}, \operatorname{iec}_{j 2}\right)+\cdots \cdots \cdots+D_{T}\left(i e c_{i T}, i e c_{j T}\right)}{T}}
$$

Con $0<D_{t}\left(\right.$ iec $\left._{i t}, i e c_{j t}\right)<4$, la distancia $d$ a lo largo de $T$ toma valores entre 0 y $2\left(0 \leq d_{\text {ind }} \leq 2\right)$.

\section{Taxonomía de los industriales según sus expectativas}

Si bien, en teoría los indicadores de expectativas podrían adoptar cualquier valor en el rango [-1,1], sus valores no se distribuyeron uniformemente, concentrán-

5 Los datos de las encuestas fueron facilitados de forma innominada (solo con la identificación de la rama), por lo que se desconoce el peso de cada empresa en la rama. 
dose en torno de la media $(-0,038)$. Una aproximación a cómo se distribuyeron las respuestas en todo el periodo lo muestra la figura 1.

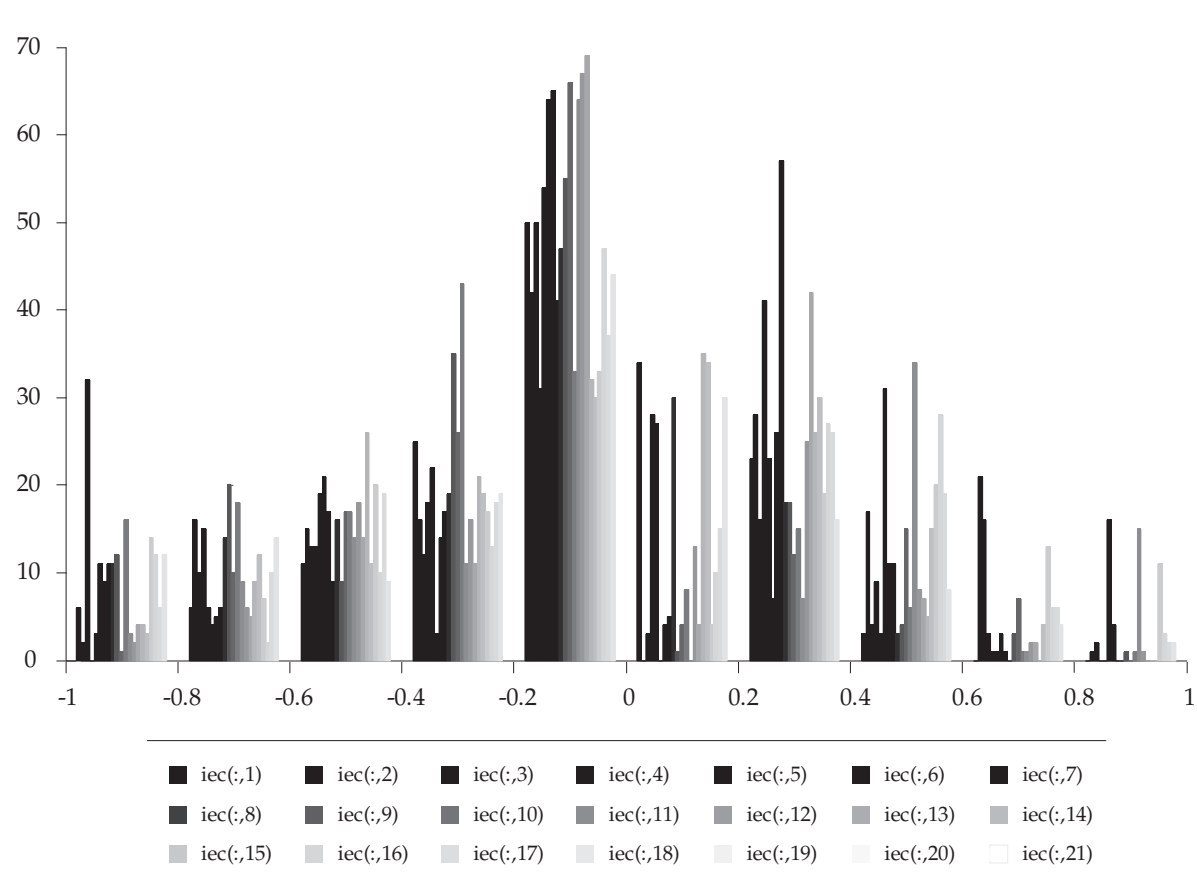

Figura 1. Histograma de expectativas por rama (i: 1-21). Enero 1998 - junio 2011

Fuente: estimaciones propias sobre la base de datos CIU.

Sobre la base de las distancias calculadas según la ecuación (5), se identificaron las conexiones más relevantes entre las ramas industriales, respecto de sus expectativas sobre la economía nacional. A partir de estas conexiones, se estimó el árbol de expansión mínima $\left(\mathrm{MST}_{\mathrm{iec}}\right)$ y las distancias ultramétricas que dan lugar al árbol jerárquico $\left(\mathrm{HT}_{\mathrm{iec}}\right)$ en los que se apoya el análisis empírico. ${ }^{6}$

Por construcción, la máxima distancia teórica entre las ramas es 2 y la mínima 0 . No obstante, la distancia máxima observada en el periodo fue sensiblemente inferior, 0,6734, lo cual sugiere que existe una razonable homogeneidad entre las ramas respecto de las expectativas que manifiestan.

Sobre la base de las 441 distancias calculadas (correspondientes a las conexiones entre las 21 ramas industriales, incluida la distancia de una rama respecto

6 Las series de expectativas, así como los programas aquí empleados están desarrollados en Matlab R2008a y están disponibles a solicitud al autor. 
de sí misma), se identificaron las conexiones más relevantes $(n-1$ conexiones, 20 en este caso). A partir de estas, se construyó el árbol de expansión mínima MST que, como se explicó, ofrece un arreglo de ramas con las conexiones más relevantes, figura $2 .{ }^{7}$ Estas representan los caminos de mínima distancia entre los nodos (vértices) del árbol.

El diámetro del MST, que ofrece una medida de la cohesión entre las ramas, fue de 6,02 en un máximo teórico de 40, lo que sugiere que la interconexión es relativamente significativa.

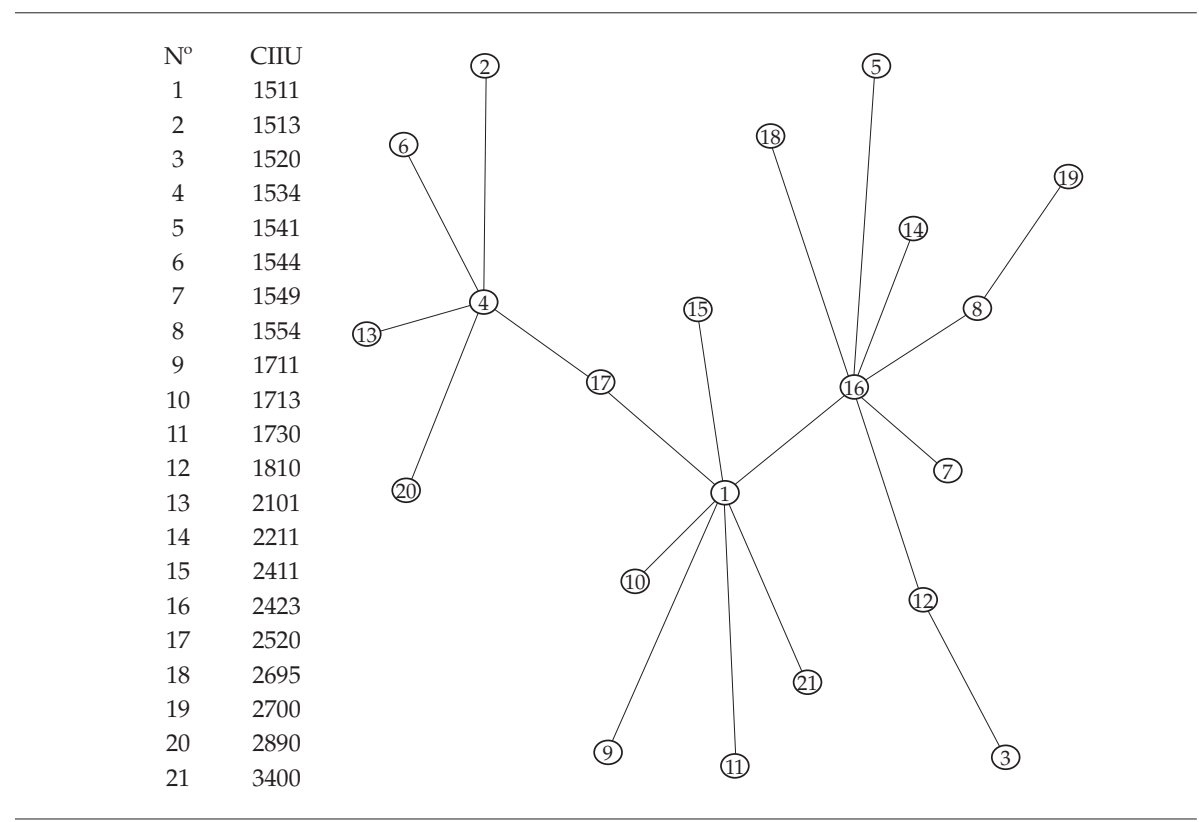

Figura 2. Árbol de expansión mínima de ramas industriales respecto de expectativas sobre la economía

Fuente: elaboración propia sobre información de CIU. Procesamientos en Matlab R2008a.

Nota: la descripción de las ramas se presenta en el anexo 2.

En la figura 2 se pueden identificar las ramas más conectadas y, en particular, las que se ubican en las posiciones centrales del árbol. Estas últimas actuarían como núcleos a nivel de la industria, ubicándose en el centro de un número significativo de sectores con las cuales mantiene un comportamiento cercano. Este árbol de distancias mínimas puede ser interpretado en el sentido que propone Potts (2000), como una red de agentes complejos y parcialmente especializados

7 Para una representación gráfica más clara del MST, las ramas o vértices del árbol no se encuentran ponderadas de acuerdo con las distancias entre ramas. 
donde algunos ocupan los nodos y otros se posicionan alrededor de estos. Los nodos son las ramas que mantienen las menores distancias en cuanto a sus expectativas sobre la economía con todas las demás, aglomerando a un subconjunto de ellas. En este sentido, podrían ser consideradas líderes en la formación de opinión, más precisamente de expectativas sobre el futuro de la economía.

En las posiciones centrales del árbol se ubicaron las ramas frigoríficos, laboratorios farmacéuticos y molinos arroceros. Algunas medidas de centralidad pueden ayudar a dimensionar el lugar que ocupan en la red de conexiones. Una de ellas es el grado de centralidad (número de conexiones directas). El grado de centralidad de los frigoríficos de los laboratorios farmacéuticos es 7 y el de los molinos arroceros, 5. Una segunda medida es la de proximidad central, definida como el número posible de conexiones (20), respecto de la distancia del nodo, en relación con los demás individuos. ${ }^{8}$ En este caso, la centralidad de frigoríficos aparece como netamente superior sobre las demás $(0,95$, siendo 0,48 la de laboratorios farmacéuticos y 0,34 la de molinos arroceros). Incluso, en este caso la centralidad de la rama 2520 (productos de plástico) supera levemente a la de molinos arroceros (llega a 0,37$).{ }^{9}$

Los sectores mencionados tienen un peso significativo en la producción industrial de Uruguay, salvo los laboratorios farmacéuticos que producen para el mercado doméstico bienes sustitutos de importaciones de su pauta exportadora. En cualquier caso, se trata de ramas muy expuestas a la competencia internacional, con presencia de empresas de gran tamaño.

El árbol jerárquico de la figura 3 muestra que, a lo largo del periodo analizado, las 21 ramas se distribuyen en 8 grupos. Por un lado, un primer gran grupo comprende 14 ramas, que se distingue de los 7 restantes que corresponden a ramas aisladas. ${ }^{10}$

Los cuadros resaltados de la figura 3 identifican el primer gran grupo formado por las siguientes ramas: molinos arroceros, frigoríficos, laboratorios, fabricación de productos plásticos, químicas, panificadoras, fabricación de bebidas sin alcohol, hilandería y tejeduría, vestimenta y tejidos de punto, imprentas, automotriz, industrias básicas de hierro y acero, otros productos de metal. Dentro de este grupo de industrias con similar comportamiento, en cuanto a sus expectativas,

8 Proximidad central $=\operatorname{Pr} C=(n-1) / \sum_{j} l(i, j)$.

9 Esto mismo ocurre cuando se toman otras medidas de centralidad alternativas como la de centralidad decreciente $C_{i}^{d}(g)=\sum_{i \neq j} \delta^{l(i, j)}$ cuando el factor de decrecimiento $\delta$ es superior a 0,33 .

${ }^{10}$ La regla de detención para establecer el número de clusters responde al criterio de pseudoF, al $10 \%$ de significación. $p s e u d o F_{0.1}(1,13)=3.616$, C.V. $=3.136, p s e u d o F_{0.1}(1,12)=2.425$, C.V. $=3.177$. 
podría tener lugar el intercambio de información, conocimientos y percepciones, en el sentido que menciona Potts (2000).

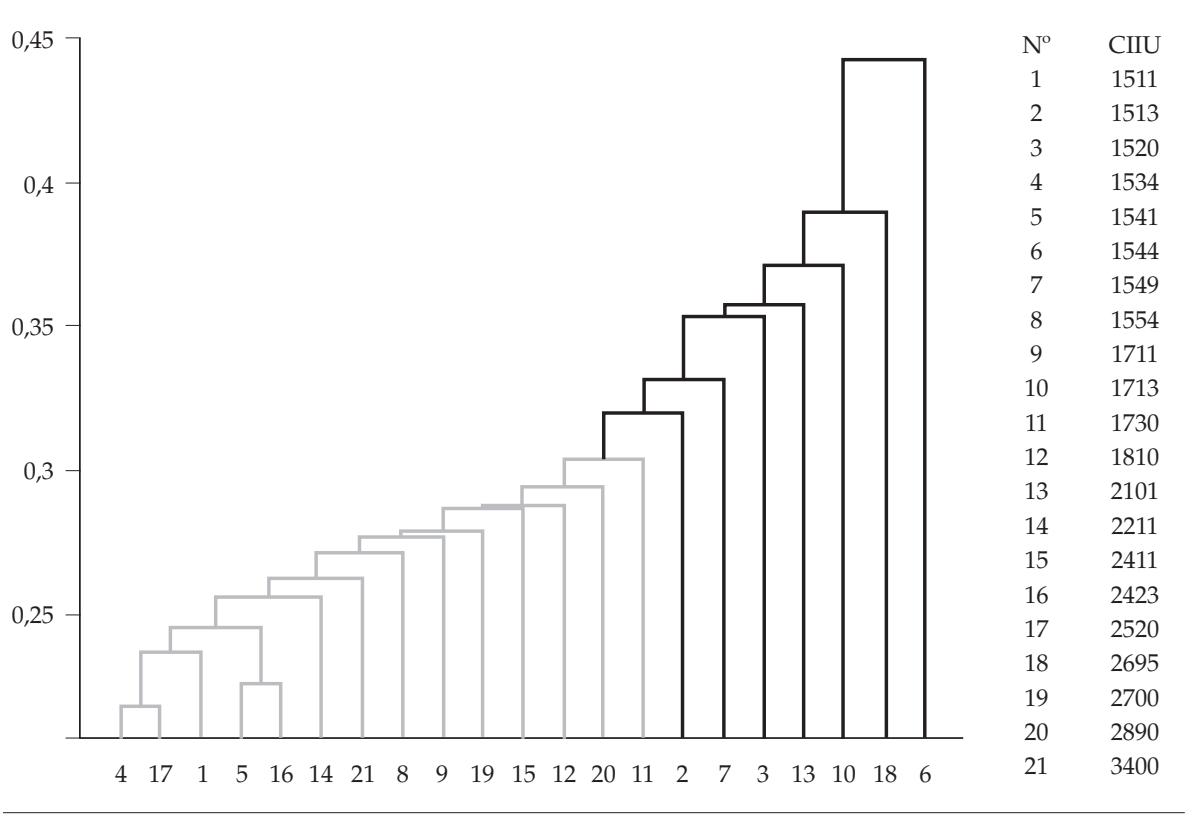

Figura 3. Árbol jerárquico de ramas industriales respecto de expectativas sobre la economía

Fuente: elaboración propia sobre información de CIU. Procesamientos en Matlab R2008a.

Dentro de este gran conglomerado de ramas industriales con similar comportamiento, en cuanto a sus expectativas sobre la economía, no se identifican subgrupos de dos casos puntuales. Pueden distinguirse dos conductas diferenciadas. Por un lado, el de las primeras 5 ramas, claramente más compactas, donde se ubican los únicos dos casos puntuales de subgrupos, y que comprende a aquellas identificadas como núcleos o nodos en el MST. Por otro, el de las restantes 9 ramas, menos cohesionadas en expectativas y que no forman subgrupos entre sí. Los sectores que no están en ese grupo de 14 ramas se encuentran aislados y no forman grupos entre ellos. La relativa debilidad de los eslabonamientos industriales (Laens \& Osimani, 2000) ${ }^{11}$ en Uruguay y la reducida cooperación inter-empresarial (Torello \& Snoeck, 1998) probablemente expliquen este relativo aislamiento.

11 Estas autoras clasifican a las ramas industriales de acuerdo con su especialización productiva e inserción comercial internacional en cuatro grupos: Exportadoras, Sustitutas de Importaciones, de Comercio Intra-rama y de Bajo Comercio. 
El cluster de las catorce ramas más compactas representa el $41 \%$ del VBP industrial (exceptuando a la refinería de petróleo), ${ }_{12}^{12}$ y se caracteriza por una importante presencia de ramas exportadoras ${ }^{13} \mathrm{y}$ de industrias que fundamentalmente elaboran manufacturas sustitutas de importaciones (siguiendo la clasificación de Laens \& Osimani, 2000). ${ }^{14}$ Es decir, industrias con una fuerte exposición a la competencia internacional, tanto por ser proveedoras de los mercados externos o por competir con productos del exterior en el mercado local. Este primer gran grupo más cohesionado se ubica en media a la izquierda del promedio general, en cuanto a expectativas, es decir, tienden a ser más pesimistas que el resto (en el promedio del periodo).

Las ramas más aisladas en sus expectativas (fideerías, lavaderos y fábricas de tops, fabricación de cemento, pulpa de madera, papel y cartón, lácteos, elaboración y conservación de frutas, legumbres y hortalizas y otros productos alimenticios) en conjunto explican algo más de $20 \%$ de la producción industrial nacional (sin refinería). Aquí, el perfil de inserción comercial de los sectores es más heterogéneo, con presencia tanto de industrias que producen principalmente para el mercado externo, como de otras que destinan sus ventas a la región o al mercado local.

\section{Distancias, clusters y contexto macroeconómico}

Las expectativas empresariales han sido señaladas simultáneamente como causa y consecuencia del contexto macroeconómico. Ha sido largamente documentado en la literatura económica que las expectativas constituyen un factor relevante en la determinación de los ciclos (desde las teorías clásicas, neoclásicas, keynesianas, neokeynesianas, etc.). Investigaciones recientes aportan evidencia de que las percepciones de los agentes sobre los fundamentos económicos actuales y futuros son capaces de generar correlaciones positivas entre las principales variables macroeconómicas (por citar algunos, Beaudry \& Portier, 2005, 2006, 2007). A su vez, el propio contexto económico afecta el ánimo de los empresarios, lo que influye en sus percepciones y expectativas futuras. Como se explicó

12 Corresponde al año 2008, última información disponible a la fecha.

13 Las ramas exportadoras incluidas en la muestra explican en su conjunto más del $75 \%$ de la producción del total de las industrias exportadoras.

14 Siguiendo la clasificación que propone Bittencourt (2011) en la publicación de ANII, (IV Encuesta de Innovación en la Industria 2007-2009), se trata de industrias fuertemente exportadoras, procesadoras de insumos primarios nacionales (frigoríficos y molinos arroceros) y de industrias moderadamente exportadoras, procesadores de insumos industriales importados (laboratorios farmacéuticos). 
antes, la complejidad del entorno económico - la presencia de incertidumbre, asimetrías de información, etc.- influye en la formación de expectativas.

Durante el periodo bajo estudio, la economía uruguaya pasó por contextos macroeconómicos de diverso signo, ciclos favorables de fuerte expansión, así como crisis profundas. A su vez, experimentó transformaciones estructurales que pudieron afectar el comportamiento empresarial del sector, implementación de una reforma tributaria, intensificación de la inversión extranjera en el sector transable, entre los más destacados.

En la figura 4 es posible advertir las fases bien diferenciadas del ciclo macroeconómico uruguayo antes referidas. ${ }^{15}$ El lapso comprendido entre el inicio de la muestra (enero de 1998 y comienzos de 1999) se caracterizó por una etapa de expansión que comienza a desacelerarse y pasa a ser francamente recesiva a principios de 2001. El valle de actividad se sitúa entre diciembre de 2002 y marzo de 2003. A partir de entonces, la economía comienza a reactivarse y afianza y profundiza su recuperación a mediados del año 2005. A partir de entonces, la actividad transita por una fase favorable del ciclo, si bien se desacelera y modera sobre fines del año 2009, incluso pasa por una muy breve etapa en la que crece por debajo de su nivel de crecimiento tendencial.

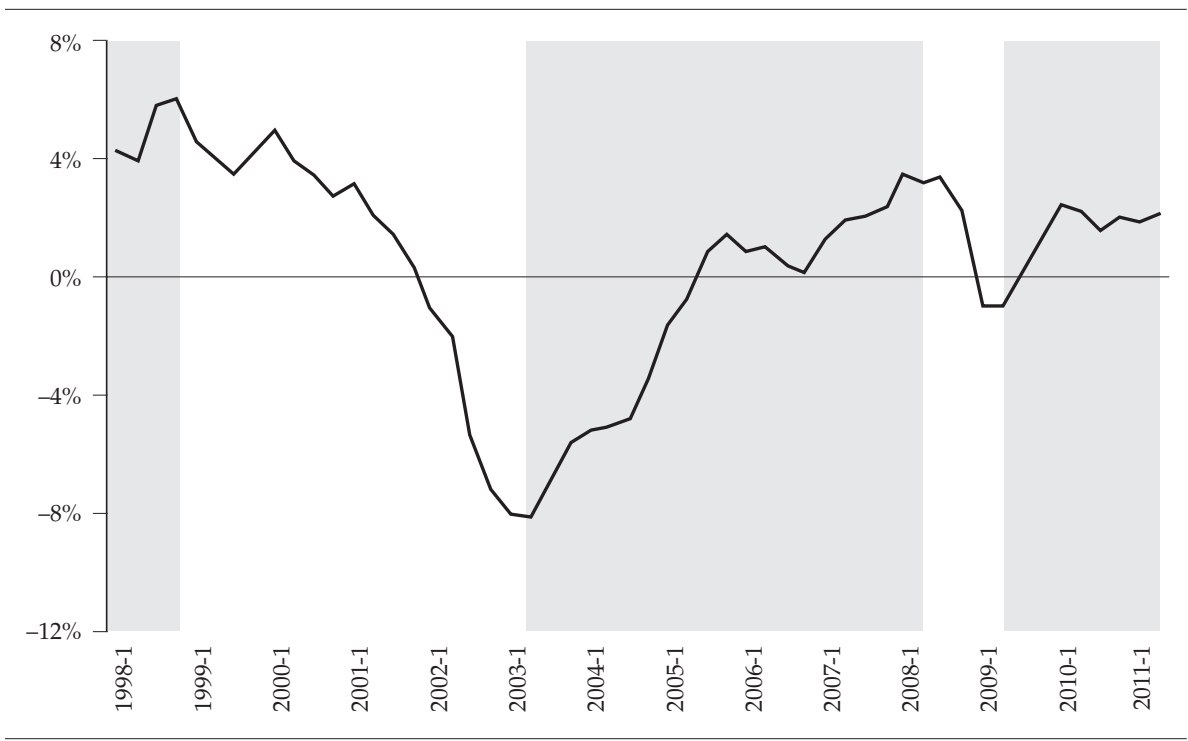

Figura 4. Ciclo del Producto Interno Bruto uruguayo. (En \% de la tendencia) Fuente: estimaciones propias (STAMP) sobre la base de datos de BCU.

15 El componente cíclico del PIB fue extraído por medio del filtro de Kalman y la estimación de un modelo estructural (siguiendo la propuesta de Harvey, 1989), empleando el software STAMP 8.30 (C) S. J. Koopman y A. C. Harvey, 1995-2010. 
Con el objetivo de encontrar evidencia que permitiera confirmar o rechazar la hipótesis respecto de la existencia de una asociación entre el ciclo económico y la forma en que se agrupan los sectores industriales respecto de sus expectativas, se dividió el periodo de catorce años en lapsos donde claramente se distinguieran las etapas de aceleración y crecimiento (oscuros de la figura) de las de depresión y desaceleración (claros).

Los resultados mostraron que en las fases cíclicas de recuperación y aceleración de la actividad se conforma una menor cantidad de clusters, al contrario de las recesivas y de desaceleración (6 y 8, respectivamente).${ }^{16}$ En las primeras (figura 5) se encontró un gran grupo de opinión similar al hallado para el periodo total. En este caso, compuesto por 16 ramas que en su mayoría no forman grupos entre sí (salvo casos puntuales). Los restantes 5 grupos constituyen 5 ramas aisladas.

En los periodos de desaceleración y recesión se hallaron 8 grupos. Un primer cluster de 6 ramas industriales, que es el que presenta menores distancias entre sí. Se identificaron dos clusters adicionales de 5 integrantes cada uno, que presentan distancias medias en la comparación. Aisladas, aparecen las ramas que conforman los restantes 5 grupos. Nótese que en el grupo más cohesionado es en el que se encuentran las ramas industriales identificadas como sectores nodos en el periodo completo: frigoríficos, molinos arroceros y laboratorios farmacéuticos.

De la simple observación gráfica de los árboles jerárquicos, se puede percibir que en los contextos más beneficiosos las distancias máximas entre las ramas industriales se encuentran en el orden de magnitud de las mínimas distancias observadas en los periodos menos favorables. La comparación de los diámetros de los árboles de expansión mínima, correspondientes a ambos contextos, ratifica esta observación. ${ }^{17}$ En momentos de expansión cíclica, el diámetro del MST (que representa la suma de las distancias mínimas entre las ramas) es significativamente inferior al de los periodos de contracción del ciclo 4,36 y 7,94, respectivamente.

16 El criterio de detención para determinar el número de clusters fue el $p s e u d o F$, al $10 \%$ de significación. Recuperación y aceleración: $p s e u d o F_{0.1}(1,15)=5.237, C . V .=3073$, pseudoF $F_{0.1}(1,14)$ $=3476, \mathrm{C} . \mathrm{V} .=3102$. Recesión y desaceleración: $p s e u d o F_{0.1}(1,13)=53.091, \mathrm{C} . \mathrm{V} .=4667$, pseu$d o F_{0.1}(1,12)=0,486$, C.V. $=4717$.

17 Como se explicó, el diámetro de expansión mínima constituye una medida de cohesión entre las ramas. 


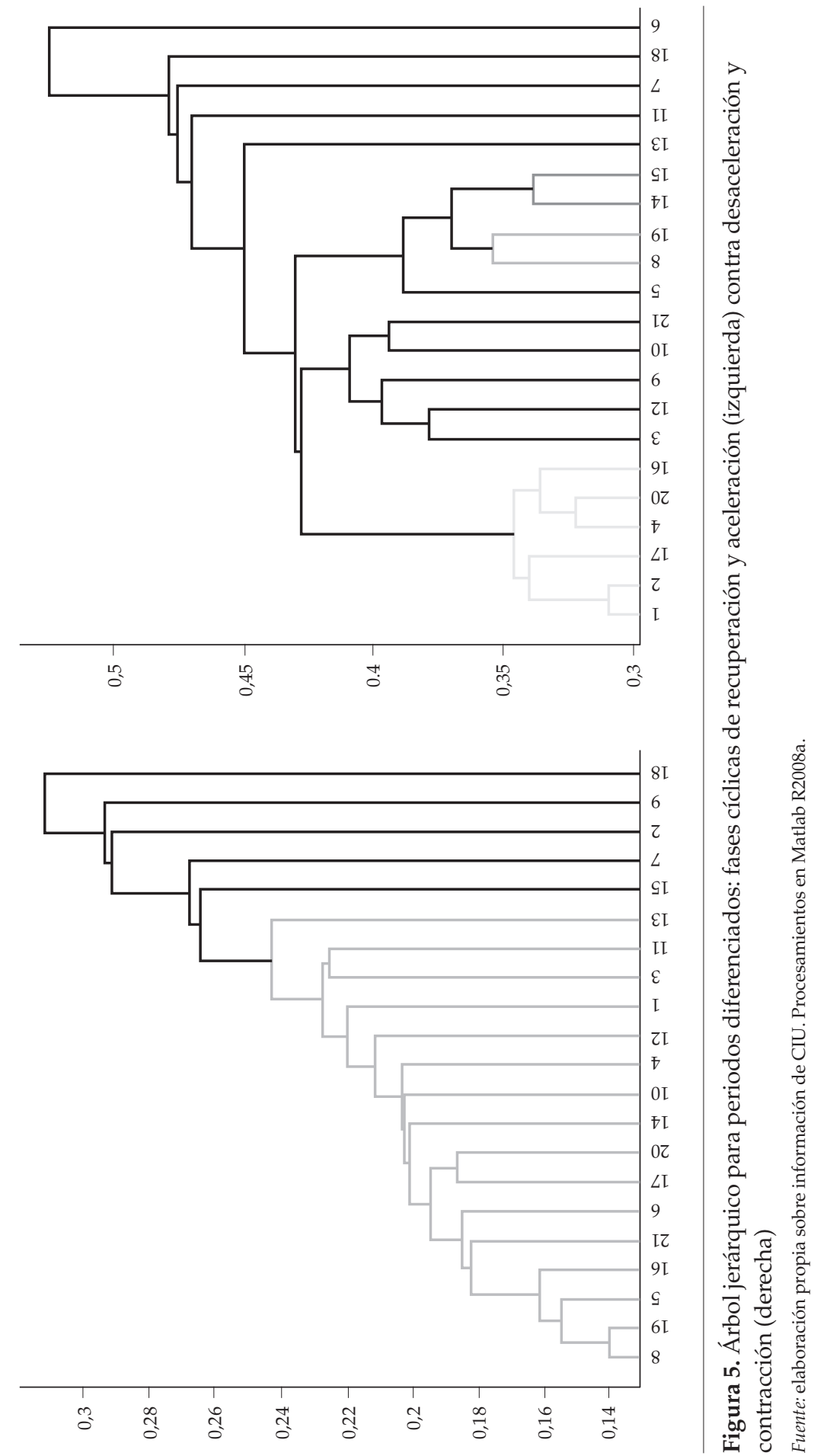


En suma, esta primera identificación de diferencias en la conformación de grupos, en cuanto a expectativas en contextos macroeconómicos de distinto signo, parece sugerir que cuando este empeora (porque el crecimiento se desacelera o la actividad cae) la dispersión y heterogeneidad entre las expectativas de los empresarios industriales aumenta. En tales circunstancias, la incertidumbre respecto del futuro crece y es probable que no todos los empresarios y sectores tengan igual acceso a la información macroeconómica más relevante, ni los afecte ni procesen de igual forma. No obstante, en estos contextos, aun cuando las distancias entre ramas respecto de sus opiniones sobre el futuro aumentan, es posible detectar grupos más definidos, contrariamente a lo que sucede cuando las señales desde la macroeconomía son de mejora. Si bien, en esos momentos los sectores parecen estar más de acuerdo con sus expectativas (las distancias son menores), no es posible identificar clusters o grupos de opinión definidos (recuérdese que este mismo patrón se encontraba en el análisis del periodo completo).

Para verificar esta primera evidencia se aplicó una estrategia alternativa, que implicó dinamizar el análisis empírico, sin necesidad de diferenciar las fases a priori. Se consideraron ventanas móviles de doce meses de amplitud para todo el periodo de referencia, y en ellas se calculó la suma de distancias mínimas entre sectores, respecto de sus expectativas. ${ }^{18}$ Esta serie de distancias mínimas móviles de 12 meses fue comparada con la tendencia subyacente ${ }^{19}$ de la producción industrial. ${ }^{20}$ En la figura 6 se representan ambas series.

El análisis de las correlaciones cruzadas de las distancias entre sectores respecto de su percepción a futuro de la economía y la producción industrial, presentó una correlación negativa significativa. A mayor distancia (menor cohesión de expectativas) peor desempeño productivo. Dicha correlación es significativa en los rezagos 5, 6 y 7 cuando se considera la tendencia subyacente en $t$ y las distancias rezagadas, y en el sexto rezago cuando se consideran ambas variables en diferencias. ${ }^{21}$ Esto indica, por tanto, que el grado de conexión entre

18 Se consideraron las distancias centradas (en el mes $t+6$ ).

${ }^{19}$ La tendencia subyacente brinda una señal suave de la evolución de la producción industrial (Espasa \& Cancelo, 1993). Se calcula a partir de la variación promedio semestral, centrado del componente de tendencia ciclo de la serie de referencia: $T_{6}^{6}=\left(\frac{\sum_{t+6}^{t+1} I V F i}{\sum_{t}^{t-5} I V F i}-1\right) * 100$.

${ }^{20}$ Se trabajó con la producción industrial (IVF de la industria manufacturera, INE) y no con el componente cíclico del PIB, debido a que la frecuencia requerida de los datos era mensual, a causa del número de distancias móviles entre expectativas que pudo calcularse. Debido a la ausencia de algunos datos en la EMI al inicio de la muestra, las distancias móviles anuales pudieron ser calculadas solo a partir de noviembre de 2001.

21 En el anexo se incluyen las salidas econométricas correspondientes. En función de que la 
las expectativas de los empresarios anticiparía el desempeño posterior de la industria en hasta seis meses.

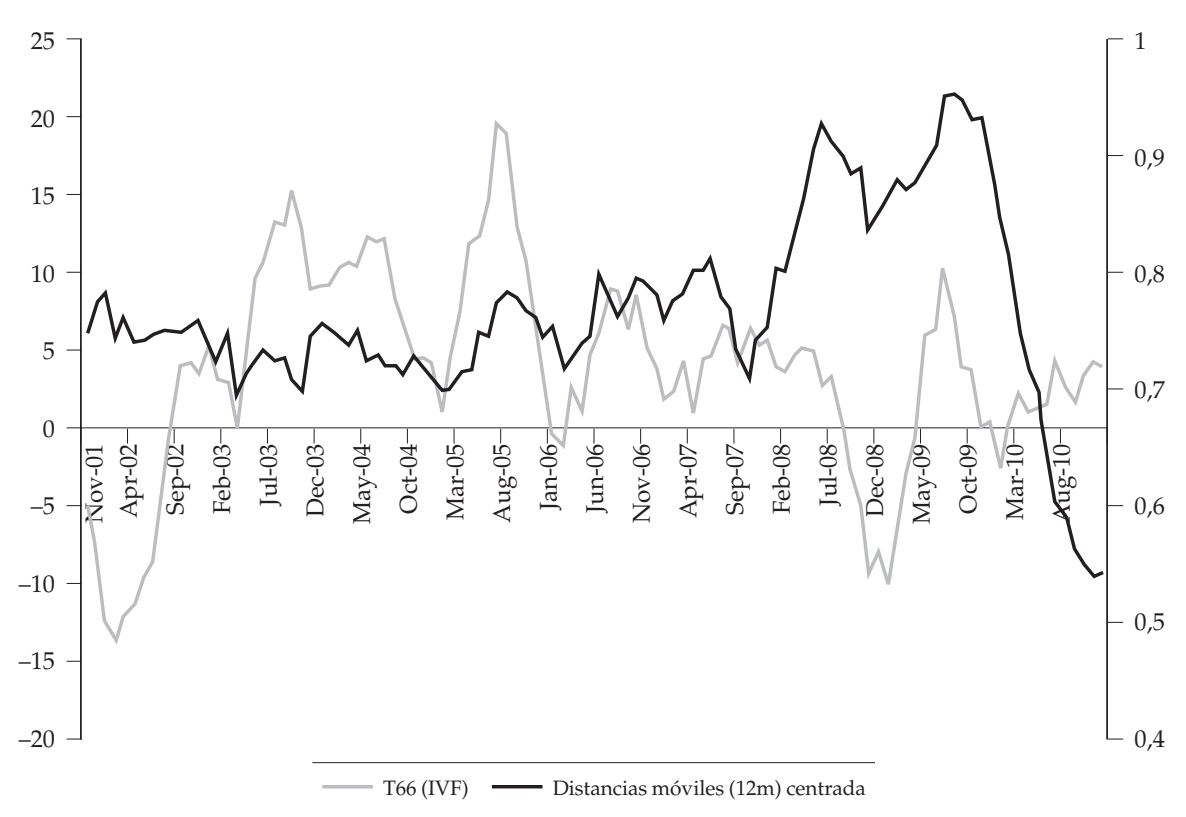

Figura 6. Distancias mínimas móviles (ventanas de 12 meses) y Tendencia subyacente $\left(T_{6}^{6} I V F\right)$ de la producción industrial (sin refinería)

Fuente: elaboración propia sobre información de CIU e INE. Procesamientos en Matlab R2008a y EViews 8.

El hecho de que el aumento de la dispersión en las expectativas entre los sectores industriales sea una señal que anticipe el empeoramiento del desempeño del sector, se encuentra en línea con la regla de incertidumbre que formula Grandmont (1998). Recuérdese que este autor plantea que cuando los agentes enfrentan algún tipo de incertidumbre acerca de la dinámica del sistema económico o social, el proceso de aprendizaje puede a su vez profundizar la incertidumbre, lo que genera inestabilidad local y profecías autocumplidas.

simple evidencia de correlación podría ser débil a favor de la asociación entre las variables, se puso a prueba la existencia de una relación de cointegración entre ellas (procedimiento Johansen). El contraste dio como resultado la existencia de una relación de equilibrio, los resultados del contraste se presentan en el anexo. 


\section{Conclusiones y consideraciones finales}

Recientes estudios desarrollados desde un enfoque macroeconómico o de agrupamientos industriales dan la pauta de la importancia de las interacciones sectoriales en la transmisión de los shocks sobre las expectativas empresariales y la actividad industrial y global en Uruguay. La presente investigación contribuye con una nueva evidencia al estudio de las expectativas empresariales, en la rama industrial, desde un enfoque empírico que no limita la identificación de los vínculos y procesos. Precisamente, la combinación de esta metodología empírica y el objeto de estudio (basada en la exploración de algoritmos de agrupación mediante una única conexión con el vecino más cercano, aplicada a series temporales de expectativas empresariales) es el aporte novedoso de esta investigación. Si bien se cuenta con numerosos estudios empíricos que aplican a diversas temáticas esta combinación de metodologías estadísticas, ninguno se conoce aplicado a indicadores de expectativas (ni por tanto al análisis aplicado a expectativas empresariales en la economía uruguaya).

Los resultados encontrados confirman algunos de los hallados en estudios previos desde enfoques macro (Lanzilotta, 2006). Permiten corroborar el vínculo entre las expectativas de los agentes y la dinámica de la actividad económica, lo que brinda evidencia de que la cohesión de las ramas industriales respecto de sus expectativas tiene una correlación positiva con el crecimiento de la producción industrial (anticipando su evolución cerca de un semestre). La dispersión de expectativas en contextos de depresión posiblemente se ligue a la mayor incertidumbre y a la heterogénea disponibilidad de información (asociada con los costos de adquirirla) que es más evidente en esos momentos. A su vez, como sugiere Grandmont (1998), el propio proceso de aprendizaje en contextos de incertidumbre puede profundizar la inestabilidad y llevar a situaciones de profecías autocumplidas. Cabe señalar, no obstante, que la observación del comportamiento en las ramas no permite corroborar la hipótesis de conducta de manada por parte de los industriales.

Por otra parte, esta investigación identifica el árbol de distancias mínimas o de red de conexiones, en el sentido que propone Potts (2000), compuesta por agentes complejos y parcialmente especializados donde algunos ocupan los nodos y otros se posicionan alrededor de estos. Muestra el rol central de las industrias de perfil transable en el proceso de trasmisión de los shocks de expectativas, al identificar un gran grupo de opinión mayormente compuesto por industrias de mucho peso en la producción manufacturera y muy expuesta a la competencia internacional. Como tales, con acceso a información amplia y completa sobre el contexto macroeconómico e internacional relevante. Dentro de ese grupo de industrias, tendría lugar el intercambio de información, conoci- 
mientos y percepciones, en el sentido que menciona Potts (2000). Se identifica, en particular, a los frigoríficos, molinos arroceros y a los laboratorios farmacéuticos como las industrias que consecuentemente forman parte un grupo de opinión relativamente cohesionado.

Si bien esta investigación no analiza causalidades, de la asociación de los dos principales resultados, se pueden derivar algunas implicaciones para la política económica de interés. El aumento de la dispersión o distancia entre expectativas, dentro de los sectores industriales en las fases cíclicas de desaceleración o recesión, son problemas a los que podría apuntar la política económica. Tómese en cuenta que, de acuerdo con este estudio, el aumento de la dispersión de opiniones, respecto al futuro entre las ramas industriales, anticipa la desaceleración y caída de la producción del sector. En esos contextos, la identificación de las ramas núcleo que conglomeran en su entorno a otras ofrece una vía eficiente para afectar las expectativas empresariales selectivamente con el objetivo de revertir el deterioro de las condiciones económicas y evitar profundizar la incertidumbre y situaciones de profecías autocumplidas (en el sentido de lo sugerido por Grandmont, 1998).

Esta investigación deja planteados algunos interrogantes, entre los que se destaca el referente a los factores que determinan estas expectativas. Futuros estudios desde ópticas tanto micro como macroeconómicos podrán abordar este interrogante, del que se extraerán implicaciones de sumo interés para la política económica.

\section{Referencias}

Alfarano, S., \& Milakovic, M. (2010). Identification of Interaction Effects in Survey Expectations: A Cautionary Note. BERG Working Paper Series on Government and Growth, 75.

Arestis, P. (1996). Post-Keynesian economics: towards coherence. Cambridge Journal of Economics, 20, 111-135.

Batchelor, R. A. (1982). Expectations, output and inflation: the European experience. European Economic Review, 17(1), 1-25.

Berk, J. M. (1999). Measuring inflation expectations: a survey data approach. Applied Economics, 31, 1467-1480.

Bittencourt, G. (2011). IV Encuesta de Innovación en la Industria Uruguaya (2007-2009). Colección de Indicadores y Estudios Agencia Nacional de la Innovación, 6.

Borraz, F., \& Gianelli, D. (2010). A Behavior Analysis of the BCU Inflation Expectation Survey. MPRA Paper 27713. 
Brida, J. G., London, S., \& Risso, W. A. (2010) Economic performance clubs in the Americas: 1955-2003. CEPAL Review, 101, 39-57.

Brida, J. G., Matesanz, D., \& Risso, W. A. (2009). Estructura jerárquica y dinámica en los mercados cambiarios latinoamericanos. Investigación Económica, LXVIII(267), 115-146.

Brida J. G., Parte L., Risso, W. A., \& Such, M. J. (2010). Hierarchical structure of the internationalized Spanish hotel industry. Tourism Management, 31(1), 57-73.

Brida, J. G., Puchet A. M., \& Punzo, L. (2003). Coding economic dynamics to represent regime dynamics. A teach-yourself exercise. Structural Change and Economic Dynamics, 14, 133-157.

Brida, J. G., \& Punzo, L. F. (2003). Symbolic Time Series Analysis and Dynamic Regimes. Structural Change and Economic Dynamics, 14(2), 159-183.

Brida, J. G., \& Risso, W. A. (2007). Dynamics and Structure of the Main Italian Companies. International Journal of Modern Physics C [Computational Physics and Physical Computation], 18(11), 1783-1793.

Carabelli, A. M. (1988). On Keynes's Method. London: Macmillan.

Chan-Lee, J. (1980). A review of recent work in the area of inflationary expectations. Review of World Economics, 116(1), 45-86.

Common, M. (1985). Testing for rational expectations with qualitative survey data. Manchester School of Economic and Social Statistics, 53(2), 138-148.

Davis, J. B. (1993). Keynes's Philosophical Development. Cambridge: Cambridge University Press.

Espasa, A., \& Cancelo, J. R. (1993). Métodos Cuantitativos para el Análisis de la Coyuntura Económica. Madrid: Alianza Editorial.

Fung, G. (2001). A Comprehensive Overview of Basic Clustering Algorithms. Recuperado de http:/ / www.cs.csi.cuny.edu.

Grandmont, J. M. (1998). Expectations Formation and Stability of Large Socioeconomic Systems. Econometrica, 66, 741-781.

Hanssens, D., \& Vanden A., P. (1987). A time-series study of the formation and predictive performance of EEC production survey expectations. Journal of Business and Economic Statistics, 5, 507-519.

Kahneman, D., Slovic, P., \& Tversky, A. (Eds.) (1982). Judgment under Uncertainty: Heuristics and Biases. Cambridge: Cambridge University Press.

Kangasniemi, J., Kangassalo P., \& Takala K. (2010). What Affects the Views about the Economic Sentiment? Evidence from the Consumer and Manufacturing Surveys in Finland. 30a Conferencia CIRET, New York.

Kangasniemi, J., \& Takala, K. (2012). The Role of Expectation Surprises in Production Decisions Evidence from the Finnish Manufacturing Survey. 31a Conferencia CIRET, Viena. 
Kauppi, E., Lassila J., \& Terasvirta, T. (1996). Short-term forecasting of industrial production with business survey data: Experience from Finland's great depression 1990-1993. International Journal of Forecasting, 12, 373-381.

Keynes, J. M. (1936). The General Theory of Employment, Interest and Money. Macmillan, London. Collected Writings vol VII. London: Macmillan, for the Royal Economic Society, 1973. Citado en: Dow, Sh. (2011), Keynes on Knowledge, Expectations and Rationality. En Frydman R. \& Phelps E. (Eds.), Rethinking Expectations: the Way Forward for Macroeconomics (pp. 112-129) Princeton University Press.

Kruskal, J. B. (1956). On the Shortest Spanning Subtree of a Graph and the Traveling Salesman Problem. Proceedings of the American Mathematical Society, 7(1), 48-50.

Laens, S., \& Osimani, R. (2000). Patrones de comercio y desempeño exportador, el caso de Uruguay en los noventa. Montevideo: Centro de Investigaciones Económica.

Lanzilotta, B. (2006). Aporte de los índices líderes de actividad económica al análisis de la coyuntura y la predicción macroeconómica en Uruguay. (Tesis de Maestría, Universidad de la República, Montevideo, Uruguay).

Lanzilotta, B. (2014). Expectativas y producción industrial en el Uruguay: interdependencia sectorial y tendencias comunes. Revista CEPAL 113, 93-119.

Lanzilotta, B., Fernández, A., \& Zunino, G. (2008). Evaluación de las proyecciones de analistas: la encuesta de expectativas de inflación del Banco Central. Monetaria, XXXI(1), 1- 25.

Mantegna, R. (1999). Hierarchical Structure in Financial Markets. The European Physical Journal B, 11, 193-197.

Nardo, M. (2003). The quantification of qualitative survey data: a critical assessment. Journal of Economic Surveys, 15(5), 645-668.

Nardo, M., \& Cabeza-Gutés, M. (2009). Rational expectations and the quantification of survey data: the role of measurement error. In Puttaswamaiah, K. (Edit.), Future of economic science (pp. 453-477). New Hampshire: Isle Publishing Company.

Öller, L. (1990). Forecasting the business cycle using survey data. International Journal of Forecasting, 6(4), 453-461.

Pesaran, M. H., \& Weale, M. (2006). Survey Expectations. En G. Elliot, Granger, C. \& Timmermann (Eds.) Handbook of Economic Forecasting. Vol 1. (pp. 715776). Amsterdam: North Holland.

Potts, J. (2000). Evolutionary Microeconomics and the Theory of Expectations. Discussion Papers Series 270, Australia: University of Queensland, Australia. 
Rahiala, M., \& Teräsvirta, T. (1993). Business survey data in forecasting the output of Swedish and Finnish metal and engineering industries: a Kalman Filter approach. Journal of Forecasting, 12, 255-271.

Rosser, J. B. Jr. (2001). Alternative keynesian and postkeynesian perspectives on uncertainty and expectations. Journal of Post Keynesian Economics, 23(4), 545-566.

Roy, S., Sivakumar, K., \& Wilkinson, I. (2004). Innovation Generation in Supply Chain Relationships: A Conceptual Model and Research Propositions. Journal of the Academy of Marketing Science, 32(1), 61-79.

Smith, J., \& Mcaleer, M. (1995). Alternative Procedures for Converting Qualitative Response Data to Quantitative Expectations: An Application to Australian Manufacturing. Journal of Applied Econometrics, 10, 165-185.

Sorenson, O., Rivkin, J. W., \& Fleming, L. (2006). Complexity, networks and knowledge flow. Research Policy, 35, 994-1017.

Svensson, L. (1997). Inflation Forecast Targeting: Implementing and Monitoring Inflation Targets. European Economic Review, 41(6), 1111-1146.

Thaler, R. H. (1994). The Winner's Curse: Paradoxes and Anomalies of Economic Life. Princenton: Princeton University Press.

Torello, M., \& Snoeck, M. (1998). La cooperación interempresarial en la industria uruguaya. CEPAL, Montevideo, 169.

Woodford, M. (2001). Imperfect Common Knowledge and the Effects of Monetary Policy. En P. Aghion, R. Frydman, J. Stiglitz, \& M. Woodford, (Eds.), Knowledge, Information, and Expectations in Modern Macroeconomics: In Honor of Edmund S. Phelps Princeton : Princeton University Press.

Zunino, G., Lanzilotta, B., \& Fernández, A. (2010). ¿Son racionales los pronósticos de inflación? Una discusión sobre la base de la Encuesta de expectativas del BCU. Montevideo: Centro de Investigaciones Económicas DT 07. 
254 Taxonomía y dinámica de las expectativas económicas de los empresarios industriales

\section{Anexos}

\section{Anexo 1}

Tabla A.1. Correlaciones cruzadas distancias mínimas móviles centradas y T $T_{6}^{6} I V F$ industria manufacturera

Sample: 2001M11 2010M12

Included observations: 110

Correlations are asymptotically consistent approximations

\begin{tabular}{|c|c|c|c|c|c|c|}
\hline \multicolumn{2}{|c|}{ T66IVF, D12M CENT (-i) } & \multicolumn{2}{|c|}{ T661IVF, D12M CENT (+i) } & \multirow{2}{*}{$\begin{array}{l}\mathrm{i} \\
0\end{array}$} & \multirow{2}{*}{$\begin{array}{l}\text { lag } \\
-0.149\end{array}$} & \multirow{2}{*}{$\begin{array}{l}\text { lead } \\
-0.149\end{array}$} \\
\hline$* * 1$. & । & $* * 1$. & I & & & \\
\hline$* * 1$. & I &.$*$. & । & 1 & -0.1964 & -0.1149 \\
\hline$* * 1$. & । & .1 . & I & 2 & -0.2441 & -0.0966 \\
\hline$* * * 1$. & I &.$* 1$. & । & 3 & -0.2964 & -0.0921 \\
\hline$* * * 1$. & I &.$* 1$. & I & 4 & -0.3392 & -0.1135 \\
\hline$* * * * 1$ & । &.$* 1$. & । & 5 & -0.3677 & -0.127 \\
\hline$* * * * 1$. & I &.$*$. & । & 6 & -0.3727 & -0.1388 \\
\hline$* * * * \mid$ & । & $* * 1$. & । & 7 & -0.3523 & -0.1591 \\
\hline$* * * 1$. & I & $* * 1$. & । & 8 & -0.3159 & -0.1723 \\
\hline$* * * 1$. & I & $* * 1$. & । & 9 & -0.2679 & -0.1715 \\
\hline$* * 1$. & I & $* * 1$. & । & 10 & -0.218 & -0.1505 \\
\hline$* * 1$. & I &.$* 1$. & । & 11 & -0.1639 & -0.1311 \\
\hline .1 . & I & .1 . & I & 12 & -0.1256 & -0.118 \\
\hline
\end{tabular}

Sample: 2001M11 2010M12

Included observations: 110

Correlations are asymptotically consistent approximations

\begin{tabular}{|c|c|c|c|c|c|c|}
\hline \multicolumn{2}{|c|}{ IVF), D(D12M CENT)(-i) } & \multicolumn{2}{|c|}{ D(T66IVF), D(D12M CENT)(+i) } & \multirow{2}{*}{$\frac{\mathrm{i}}{0}$} & \multirow{2}{*}{$\begin{array}{l}\text { lag } \\
0.1087\end{array}$} & \multirow{2}{*}{$\begin{array}{l}\text { lead } \\
0.1087\end{array}$} \\
\hline $.1 *$ & | & $.1 *$ & 1 & & & \\
\hline .1. & । & $.1^{* *}$ & I & 1 & -0.0035 & 0.1923 \\
\hline .1. & । &.$I^{*}$ & I & 2 & 0.037 & 0.1121 \\
\hline.$* 1$ & । &.$I^{*}$ & I & 3 & -0.0874 & 0.1252 \\
\hline.$* 1$ & । & .1. & I & 4 & -0.1252 & -0.0086 \\
\hline$* * 1$. & । & .1. & I & 5 & -0.213 & 0.0195 \\
\hline
\end{tabular}




\begin{tabular}{|c|c|c|c|c|c|c|}
\hline$* * 1$. & 1 & .1. & I & 6 & -0.2281 & -0.0169 \\
\hline.$* 1$. & 1 &.$^{*} 1$. & I & 7 & -0.147 & -0.0682 \\
\hline.$* 1$ & 1 &.$* 1$ & I & 8 & -0.094 & -0.1339 \\
\hline .1. & I & .1. & I & 9 & -0.0096 & -0.1364 \\
\hline .1. & 1 & ${ }^{*} 1$. & I & 10 & -0.0332 & -0.0606 \\
\hline.$I^{*}$ & I & .1 & I & 11 & 0.1425 & -0.0293 \\
\hline $.1^{*}$ & I &.$^{*} 1$. & I & 12 & 0.1402 & -0.091 \\
\hline
\end{tabular}

Tabla A.2. Contraste de cointegración (Johansen) [D12M_cent, $T_{6}^{6} I V F$ ind.man]

\begin{tabular}{llrrrrr}
\hline \multirow{2}{*}{\begin{tabular}{c} 
Especificación: varia- $\begin{array}{c}c \\
\text { bles, lags }\end{array}$ \\
\cline { 2 - 7 }
\end{tabular}} & $\begin{array}{c}\text { No. de } \\
\text { CE(s) }\end{array}$ & Eigenvalue & Estadístico & Prob. & Estadístico & Prob \\
\hline [T66IVF D12M_CEN] & None & 0.1853 & 25.76132 & $0.0079^{* *}$ & 21.51827 & $0.0058^{* *}$ \\
Lags 1-4 & At most 1 & 0.0396 & 4.243055 & 0.3771 & 4.243055 & 0.3771 \\
cons. restricta & & & & & & \\
Sample (adjusted): 2002M04 2010M12 & & & & & \\
\hline
\end{tabular}

Nota: Significación al 1\%**, al 5\%*.

\section{Anexo 2}

Descripción de las ramas industriales (Capítulo 5)

$\mathrm{N}^{\circ}$ CIIU3 Descripción

11511 Matanza de ganado. Preparación y conservación de carnes.

21513 Elaboración y conservación de frutas, legumbres y hortalizas.

31520 Elaboración de productos lácteos.

41534 Molinos arroceros.

51541 Elaboración de productos de panadería.

61544 Fideerías y fabricación de pastas frescas.

71549 Elaboración de otros productos alimenticios n.c.p.

81554 Fabricación de bebidas sin alcohol y aguas de mesa minerales o no.

91711 Hilandería, tejeduría y acabado de lana, algodón, sintético y fibras duras.

101713 Lavadero y fabricación de tops

$11 \quad 1730 \quad$ Fabricación de tejidos de punto.

121810 Fábricas de prendas de vestir. 
132101 Fabricación de pulpa de madera, papel y cartón.

142211 Actividades de encuadernación, impresión, edición y reproducción en papel.

152411 Fabricación de sustancias químicas básicas.

162423 Fabricación de productos farmacéuticos y medicamentos de uso médico y veterinario.

$172520 \quad$ Fabricación de productos de plástico.

182695 Fabricación artículos de cemento.

192700 Industrias básicas de hierro, acero y metales no ferrosos.

202890 Fabricación de otros productos de metal.

213400 Fabricación de vehículos automotores, carrocerías, cúpulas, repuestos, motores. 